\title{
Fractional Calculus and the Evolution of Fractal Phenomena
}

\author{
Andrea Rocco and Bruce J. West \\ Center for Nonlinear Science, University of North Texas, P.O. Box 5368, Denton, Texas 76203-5368
}

(October 29, 2018)

\begin{abstract}
It is argued that the evolution of complex phenomena ought to be described by fractional, differential, stochastic equations whose solutions have scaling properties and are therefore random, fractal functions. To support this argument we demonstrate that the fractional derivative (integral) of a generalized Weierstrass function (GWF) is another fractal function with a greater (lesser) fractal dimension. We also determine that the GWF is a solution to such a fractional differential stochastic equation of motion.
\end{abstract}

Pacs number(s): 47.53.+n, 02.30.Bi, 05.40.+j

Key Words: Fractal, Complex System, Fractional Calculus

\section{INTRODUCTION}

Significant changes in our knowledge of how to analyze nonlinear static and dynamical phenomena in the physical and biological sciences have occurred over the past thirty years 1 迎. In the physical sciences methodology has moved away from complete reliance on the tools of linear, analytic, quantitative mathematical physics towards a combination of nonlinear, numerical and qualitative techniques [3]. Not only have many of the linear analytical approaches often proved to be inadequate, but the entrenched geometry of Euclid, classically used to describe natural phenomena, has not always been adequate to the task. In the 1960s Mandelbrot began discussing a new geometry of nature, one that embraces the irregular shapes of objects such as coastlines, lighting bolts, clouds and molecular trajectories. A common feature of these objects, which Mandelbrot called fractals, is that their boundaries are so irregular that it is not easy to understand how to apply simple metrical ideas and operations to them [1].3]. Towards this end we shall consider some of the metric peculiarities of a few unusual mathematical objects, fractal functions, and discuss the possible physical implications of their evolution in time in terms of the phenomena they are used to model.

One of the defining properties of a fractal function is that it does not possess a characteristic scale length and consequently its derivatives diverge. The Weierstrass function was the first example of a function that is continuous everywhere, but is nowhere differentiable. In 1926 Richardson [5] suggested that this function, or one sharing its non-analytic properties, be used to describe the velocity field of the atmosphere, because of the apparent impossibility of modeling the irregular flow of the wind using differential equations. It was specifically this property of non-differentiability that Richardson believed captured the essential features of turbulence. This view of turbulence, rather than becoming quaint and out of date, has been demonstrated to be quite modern [6] and in fact anticipated the introduction of fractals into the description of complex phenomena [1].3.
During the same period Lévy [7] was working to establish the most general properties necessary for a process to violate the then accepted form of the Central Limit Theorem and still converge to a limit distribution. He was quite successful, establishing the class of infinitely divisible distributions, which as its name implies concerns processes whose statistical properties persist at each level of aggregation of the data, and are today called $\alpha$-stable Lévy processes. A deep connection between Lévy stable processes and the Weierstrass function was established using random walk concepts [8 10] and subsequently used to understand turbulent fluid flow [6].

It is not only macroscopic phenomena such as turbulence that suffer from the problem of not being describable by analytic functions, however. The theory of Brownian motion as formulated in 1908 by Langevin [11] has the form

$$
\frac{d u(t)}{d t}=-\lambda u(t)+F(t)
$$

where $u(t)$ is the velocity of the Brownian particle, $\lambda$ is the dissipation rate and $F(t)$ is the random influence of the bath of lighter particles (molecular impacts) on the Brownian particle. In 1909 Perrin [12] observed that the path of a Brownian particle, as seen through his microscope, is continuous but not differentiable. Therefore such a path can not be described by an analytic function. In 1942 Doob [13] proved that the velocity of the Brownian particle is discontinuous for the Ornstein-Uhlenbeck process [14] described by (11) and discusses how one constructs a stochastic differential equation to replace the more familiar differential equations of physics up to that time. In particular he replaced (传) with

$$
d u(t)=-\lambda u(t) d t+d B(t)
$$

where $d B(t)$ is the increment of a Wiener process. This new mathematics has become so accepted that we now write the Langevin equation (11), but interpret it as the Doob equation (2). A process appears random on the macroscopic level due to the separation of time scales in 
the microscopic and macroscopic processes. When such a separation exists the Langevin equation, using the interpretation of Doob, adequately describes the dynamics of the physical phenomenon. On the other hand, when this separation of time scales does not exist, ordinary statistical physics is no longer adequate to describe the phenomenon, as discussed by Grigolini et al. [15]. In particular, a lack of time-scale separation induces a fractional, stochastic, differential equation on the macroscopic level [2].

What the Weierstrass and other non-analytic functions and the Lévy distribution have in common is the lack of a differential description of the time evolution of the phenomena which they are intended to model. It was only a decade ago that it was recognized by Shlesinger et al. [6] that Lévy flights can be used to describe the velocity field of turbulent flow. The key to this understanding is that the equation of evolution of the probability density is a fractional diffusion equation whose solution is a truncated Lévy distribution [16].

Fractional diffusion equations have been used to model the evolution of stochastic phenomena with long-time memory, that is, phenomena with correlations that decay as inverse power laws rather than exponentially in time [2,16 18]. It has been known for quite some time in the economics literature that such statistical properties can be successfully described using finite difference equations in which the finite difference is of fractional order, see Hosking 19,20]. The continuum limit of such fractional difference stochastic equations are fractional differential stochastic equations,

$$
\frac{d^{\beta} u(t)}{d t^{\beta}}=F(t)
$$

where here the mathematics has been generalized to the fractional calculus to accommodate the non-analytic properties of the underlying phenomena. The evolution of the probability density associated with the velocity in (3) is a fractional partial differential equation in the phase space for the phenomenon, see for example West and Grigolini [21].

Herein we suggest that the dynamics of complex phenomena, described by fractal functions, can be expressed in terms of fractional differential equations of motion. One approach to doing this has been adopted by Nonnenmacher and colleagues [22], who generalize the traditional models of viscoelastic phenomena using fractional initial value problems. The solutions to their fractional equations of motion yield physical observables, such as the stress relaxation, that is in excellent agreement with experiment. Others have generalized the analysis of wave phenomena, starting with the diffractals of Berry [23], up to and including the fractional wave equation of Schneider and Wyss [24].

Our goal here is more modest in that we do not attempt to describe any particular phenomenon in complete de- tail. On the other hand it is more ambitious in that we demonstrate the generic result that fractal functions have fractional derivatives and therefore complex phenomena having a fractal dimension are more reasonably modeled using fractional equations of motion than they are using ordinary differential equations of motion such as given by Newton's laws.

Even sharing the universally accepted view according to which Newton's laws apply to the fundamental constituents of complex phenomena 225], this microscopic level of description is often not the natural one for the phenomena being studied. For example, one rarely uses particle dynamics to describe the viscous fluid flow in turbulence, instead one more often uses the Navier-Stokes equation to describe the behavior of the velocity field [26]. The familiar, ordinary differential equations of motion for individual particles need to be replaced with fractional differential and integral equations for the appropriate field variables. We show, using a generalized Weierstrass function (GWF), that if said function has a fractal dimension $D$ then the $\beta$-fractional derivative yields a new function of fractal dimension $D+\beta$. In a similar way the $\beta$-fractional integral of the generalized Weierstrass function yields a new function of fractal dimension $D-\beta$. Using these properties we can write the fractional differential equations of motion for the complex process of interest.

\section{GENERALIZED WEIERSTRASS FUNCTION}

The Weierstrass function was the first exemplar of a function that is continuous and non-differentiable. A number of generalizations of this fractal function have been discussed in the literature [1]2,27], the most general including random behavior. We refer to the latter as the generalized Weierstrass function (GWF) defined by Berry and Lewis [28] as the series:

$$
W(t)=\sum_{n=-\infty}^{\infty} \frac{\left(1-e^{i \gamma^{n} t}\right) e^{i \phi_{n}}}{\gamma^{(2-D) n}}
$$

where $1<D<2, \gamma>1$ and $\phi_{n}$ is an arbitrary phase. Berry and Lewis show using a combination of numerics and analysis that for these values of the parameters the GWF is continuous but not differentiable, has no characteristic scale, and almost certainly has a fractal dimension $D$ [29]. This argument was given mathematical rigor by Mauldin and Williams 30 somewhat later. Further, if the set of phases $\left\{\phi_{n}\right\}$ are uniformly distributed on the interval $(0,2 \pi)$ then $W(t)$ is a stochastic fractal function. The properties of a modified version of (4i) was considered by Falconer [27.

It is well known that (14) has a fractal graph, that is, the trail left by the GWF in $(X, t)$-space is a fractal curve. 
The fractal nature of such graphs are manifest in the power-law behavior of the correlation between measurements separated by a time interval $\tau$ [27]. Before we consider the increments in the GWF, that is, the difference in measurements separated by a time $\tau$, let us examine the scaling properties of (任)

$$
W(\gamma t)=\gamma^{2-D} W(t)
$$

which is obtained by relabeling the series index. The most general solution to the scaling equation (5) is [2, 3]

$$
W(t)=A(t) t^{\alpha}
$$

where $A(t)$ is a function periodic in the logarithm of $t$, with period $\ln \gamma$, and the power-law index is given by

$$
\alpha=2-D \text {. }
$$

Thus, we see that the dominant behavior of the GWF is a power-law growth in time, and the power-law index is determined by the fractional dimension, $D$.

It is possible to connect the expression for the fractional dimension, (7), with the slope of the correlation function of the increments in the GWF. Let us define such a correlation function in the following way

$$
C(\tau)=\left\langle|\Delta W(t, \tau)|^{2}\right\rangle_{\phi}
$$

where the increments of the GWF are defined by

$$
\Delta W(t, \tau)=W(t+\tau)-W(t)
$$

and the average, indicated by the brackets with a $\phi$ subscript in (8) is taken over an ensemble of realizations of the phases $\{\phi\}$ uniformly distributed on the interval $(0,2 \pi)$. We refer to the function (9) as the incremental GWF or IGWF. Inserting the IGWF

$$
\Delta W(t, \tau)=\sum_{n=-\infty}^{\infty} \gamma^{-(2-D) n}\left(1-e^{i \gamma^{n} \tau}\right) e^{i\left(\gamma^{n} t+\phi_{n}\right)}
$$

into the phase average in (8) and carrying out the phase average

$$
\langle(\ldots)\rangle_{\phi}=\prod_{n=-\infty}^{\infty} \int_{0}^{2 \pi} \frac{d \phi_{n}}{2 \pi}(\ldots)
$$

which for a uniform distribution of phases yields

$$
\left\langle e^{i\left(\phi_{n}-\phi_{n^{\prime}}\right)}\right\rangle_{\phi}=\delta_{n, n^{\prime}},
$$

the correlation function becomes

$$
C(\tau)=2 \sum_{n=-\infty}^{\infty} \gamma^{-2(2-D) n}\left(1-\cos \gamma^{n} \tau\right) .
$$

Note, since the correlation of the IGWF are independent of the initial time $t$, these increments are a realization of a stationary stochastic process.
The dominant behavior of the IGWF correlation function (13) is determined by the solution to the exact scaling relation

$$
C(\gamma \tau)=\gamma^{2(2-D)} C(\tau)
$$

obtained from (13) by again relabeling the series. The most general solution to (14) is

$$
C(\tau)=A(\tau) \tau^{2 \alpha}
$$

where as before $A(\tau)$ is a function periodic in the logarithm of its argument and the power-law index is given by (7) [2,3]. Note that $\alpha$ plays the same role here as the Hurst exponent, $H$, does in random walk processes. In fact if $\alpha=H=1 / 2$, then the correlation function increases linearly with time, so that the IGWF, $\Delta W$, would be a normal diffusive process with Gaussian statistics that is stationary in time.

\section{FRACTIONAL CALCULUS AND THE IGWF}

We are interested in both the fractional integral and the fractional derivative of the generalized Weierstrass function. However, in applying the fractional calculus, we take note of the fact that the GWF is not a stationary stochastic function. It is the IGWF that is stationary. Therefore we apply the fractional calculus to the stationary IGWF and thereby avoid some technical difficulties.

\section{A. Fractional integral of the IGWF}

Let us introduce the Riemann-Liouville definition of a fractional integral of order $\beta$ of the GWF:

$$
\mathcal{D}^{(-\beta)} W(t) \equiv \frac{1}{\Gamma(\beta)} \int_{-\infty}^{t} \frac{W\left(t^{\prime}\right) d t^{\prime}}{\left(t-t^{\prime}\right)^{1-\beta}},
$$

where $0<\beta<1$. We have for the fractional integral of the IGWF:

$$
\Delta W^{(-\beta)}(t, \tau) \equiv \frac{1}{\Gamma(\beta)} \int_{-\infty}^{t} \frac{d t^{\prime}}{\left(t-t^{\prime}\right)^{1-\beta}} \Delta W\left(t^{\prime}, \tau\right),
$$

so that inserting (10) into (17) yields

$$
\begin{aligned}
\Delta W^{(-\beta)}(t, \tau) \equiv & \frac{1}{\Gamma(\beta)} \sum_{n=-\infty}^{\infty} \frac{e^{i \phi_{n}}}{\gamma^{(2-D) n}}\left(1-e^{i \gamma^{n} \tau}\right) \\
& \times\left\{\int_{-\infty}^{t} \frac{d t^{\prime}}{\left(t-t^{\prime}\right)^{1-\beta}} e^{i \gamma^{n} t^{\prime}}\right\}
\end{aligned}
$$

The integral between the curly braces yields 


$$
\Gamma(\beta) \frac{e^{i \gamma^{n} t}}{\gamma^{\beta n}} e^{-i \pi \beta / 2}
$$

and therefore

$$
\Delta W^{(-\beta)}(t, \tau) \equiv e^{-i \pi \beta / 2} \sum_{n=-\infty}^{\infty} \frac{e^{i\left(\phi_{n}+\gamma^{n} t\right)}}{\gamma^{(2-D+\beta) n}}\left(1-e^{i \gamma^{n} \tau}\right) .
$$

Thus, we see that the fractional integral of the IGWF has the same form as the original IGWF. The difference between (20) and (10), up to an overall phase, is that $D \rightarrow D-\beta$. What is the proper interpretation of this shifting of the parameter value?

To answer this question, we need to go back to the GWF. We shall address this issue in the next section. For the time being, we limit ourselves to noticing that the correlation function related to $(20)$ is given by

$$
C(\tau)=2 \sum_{n=-\infty}^{\infty} \gamma^{-2(2-D+\beta) n}\left(1-\cos \gamma^{n} \tau\right)
$$

and therefore, if (20) corresponds to some difference of properly defined GWFs, by virtue of the scaling index (7), these new GWFs would have a new fractional dimension given by $D^{\prime}=D-\beta$. In the next section we shall make this argument rigorous.

\section{B. Fractional derivative of the IGWF}

The calculations in the case of the fractional derivative of the IGWF are similar to those carried out in the case of the fractional integral of the IGWF. Let us consider the Riemann-Liouville definition of the $\beta$ fractional derivative of the GWF:

$$
\mathcal{D}^{(\beta)} W(t) \equiv \frac{1}{\Gamma(1-\beta)} \frac{d}{d t} \int_{-\infty}^{t} \frac{W\left(t^{\prime}\right) d t^{\prime}}{\left(t-t^{\prime}\right)^{\beta}} .
$$

where again $0<\beta<1$. The fractional derivative of the IGWF result in

$$
\Delta W^{(\beta)}(t, \tau)=\frac{1}{\Gamma(1-\beta)} \frac{d}{d t} \int_{-\infty}^{t} \frac{d t^{\prime}}{\left(t-t^{\prime}\right)^{\beta}} \Delta W^{(\beta)}\left(t^{\prime}, \tau\right) .
$$

The expression (18) is now replaced with

$$
\begin{aligned}
\Delta W^{(\beta)}(t, \tau)= & \frac{1}{\Gamma(\beta)} \sum_{n=-\infty}^{\infty} \frac{e^{i \phi_{n}}}{\gamma^{(2-D) n}}\left(1-e^{i \gamma^{n} \tau}\right) \\
& \times\left\{\frac{d}{d t} \int_{-\infty}^{t} \frac{d t^{\prime}}{\left(t-t^{\prime}\right)^{1-\beta}} e^{i \gamma^{n} t^{\prime}}\right\}
\end{aligned}
$$

where the time derivative of the integral in curly braces is

$$
i \Gamma(1-\beta) \gamma^{\beta n} e^{i \gamma^{n} t} e^{-i \pi(\beta-1) / 2}
$$

and therefore

$$
\Delta W^{(\beta)}(t, \tau) \equiv e^{i \pi \beta / 2} \sum_{n=-\infty}^{\infty} \frac{e^{i\left(\phi_{n}+\gamma^{n} t\right)}}{\gamma^{(2-D-\beta) n}}\left(1-e^{i \gamma^{n} \tau}\right)
$$

Thus, we see that the fractional derivative of the IGWF has the same form as the original IGWF. The difference between (26) and (10), up to an overall phase, is that $D \rightarrow D+\beta$.

Again after observing that the correlation function related to (26) is

$$
C(\tau)=2 \sum_{n=-\infty}^{\infty} \gamma^{-2(2-D-\beta) n}\left(1-\cos \gamma^{n} \tau\right)
$$

we make the plausible conjecture that $D+\beta$ corresponds to the new fractional dimension, $D^{\prime \prime}$, of some properly defined GWF. Again this is made rigorous in the next section.

\section{WHAT ABOUT THE GWF?}

We now want to use the fractional integral and fractional derivative of the IGWF to determine these same operations of the GWF. To accomplish this we assume that the IGWF is a given, as are its fractional integral and fractional derivative, but the GWF remains to be determined. Using the RHS of (9) and (10) we obtain

$$
\begin{aligned}
W(t+\tau)-W(t)= & \sum_{n=-\infty}^{\infty} \frac{e^{i\left(\gamma^{n} t+\phi_{n}\right)}}{\gamma^{(2-D) n}} \\
& -\sum_{n=-\infty}^{\infty} \frac{e^{i\left(\gamma^{n}(t+\tau)+\phi_{n}\right)}}{\gamma^{(2-D) n}}
\end{aligned}
$$

from which we can define the function

$$
f(t)=W(t)+\sum_{n=-\infty}^{\infty} \frac{e^{i\left(\gamma^{n} t+\phi_{n}\right)}}{\gamma^{(2-D) n}}
$$

where $W(t)$ is assumed to be unknown, and (28) is replaced with the condition

$$
f(t+\tau)=f(t) .
$$

The series in (29) is divergent, so that in order to regularize the function we write

$$
f_{N}(t)=W_{N}(t)+\sum_{n=-N}^{N} \frac{e^{i\left(\gamma^{n} t+\phi_{n}\right)}}{\gamma^{(2-D) n}}
$$

where 


$$
\begin{aligned}
f(t) & =\lim _{N \rightarrow \infty} f_{N}(t) \\
W(t) & =\lim _{N \rightarrow \infty} W_{N}(t) .
\end{aligned}
$$

The constraint (30) is now replaced with

$$
\lim _{N \rightarrow \infty} f_{N}(t+\tau)=\lim _{N \rightarrow \infty} f_{N}(t)
$$

indicating that the regularized function is either periodic with period $\tau$ or is a constant in the limit.

We now wish to establish that knowing the IGWF uniquely determines the function $W(t)$, and this is the GWF. To accomplish this we use the constraint given by (33). We present the analysis for the case $f_{N}(t)=A_{N}$, where $A_{N}$ is a constant, so that (31) can be written

$$
W_{N}(t)=A_{N}-\sum_{n=-N}^{N} \frac{e^{i\left(\gamma^{n} t+\phi_{n}\right)}}{\gamma^{(2-D) n}} .
$$

In order to determine the constant in (34) we impose an additional constraint on the function. Since we want the function $W(t)$ to be a fractal we require that $W_{N}(t)$ satisfy the scaling condition,

$$
\lim _{N \rightarrow \infty} W_{N}(\gamma t)=\lim _{N \rightarrow \infty} b W_{N}(t)
$$

where $b$ is a constant. Note that we exclude the possibility that the function $f_{N}(t)$ is periodic, since this will not satisfy this additional requirement that the function also scales. Imposing this scaling constraint on (34) we have

$$
W_{N}(\gamma t)=A_{N}-\gamma^{(2-D)} \sum_{n=-N+1}^{N-1} \frac{e^{i\left(\gamma^{n} t+\phi_{n}\right)}}{\gamma^{(2-D) n}}
$$

so if we choose $b=\gamma^{2-D}$ we can satisfy (35) in the $N \rightarrow \infty$ limit if we also choose

$$
A_{N}=\sum_{n=-N}^{N} \frac{e^{i \theta_{n}}}{\gamma^{(2-D) n}},
$$

where $\left\{\theta_{n}\right\}$ is a set of arbitrary phases, because

$$
\lim _{N \rightarrow \infty} A_{N}=\lim _{N \rightarrow \infty} \gamma^{2-D} A_{N}
$$

Thus, the divergences that required the regularization exactly cancel in (36) with the choice of constraint (37) and we obtain the function

$$
W(t)=\lim _{N \rightarrow \infty} W_{N}(t)=\sum_{n=-\infty}^{\infty} \frac{\left(1-e^{i \gamma^{n} t}\right) e^{i \phi_{n}}}{\gamma^{(2-D) n}}
$$

which is the GWF, where we have associated the set of phases with those used earlier in the GWF.

This argument can also be applied to the fractional integral of the IGWF given by (20) so that we also have for the fractional integral of the GWF

$$
W^{(-\beta)}(t, \tau) \equiv e^{-i \pi \beta / 2} \sum_{n=-\infty}^{\infty} \frac{e^{i \phi_{n}}}{\gamma^{(2-D+\beta) n}}\left(1-e^{i \gamma^{n} t}\right) .
$$

In the same way the fractional derivative of the IGWF given by (26) implies that the fractional derivative of the GWF is

$$
W^{(\beta)}(t, \tau) \equiv e^{i \pi \beta / 2} \sum_{n=-\infty}^{\infty} \frac{e^{i \phi_{n}}}{\gamma^{(2-D-\beta) n}}\left(1-e^{i \gamma^{n} t}\right) .
$$

Thus, our earlier remarks regarding the fractional calculus applied to the IGWF apply equally well to the GWF.

\section{CONCLUSIONS}

We have determined the fractional dimension of both fractional integral and derivative of the GWF. Our result reads in these two cases:

$$
\begin{aligned}
& \operatorname{Dim}\left[\mathcal{D}^{(-\beta)} W\right]=\operatorname{Dim}[W]-\beta, \\
& \operatorname{Dim}\left[\mathcal{D}^{(\beta)} W\right]=\operatorname{Dim}[W]+\beta .
\end{aligned}
$$

This result can be easily interpreted noticing that the fractional dimension gives information about the degree of irregularity of the function under analysis. We have demonstrated that carrying out a fractional integral of the GWF means decreasing its fractional dimension and therefore smoothing the process, while carrying out a fractional derivative means increasing the fractional dimension and therefore making the process and its increments more irregular.

A related result was obtained by Kolwankar and Gangal [31,32], but required the introduction of a local fractional derivative (LFD), that is, a fractional derivative defined such that its non-local character is removed. They find that the LFD of a Weierstrass function, that is, the imaginary part of (位) with all $\phi_{n}=0$, exists up to "critical order" $2-D$ and not so for orders between $2-D$ and 1 , where $D(1<D<2)$ is the box counting dimension of the graph of the function. It is possible to show that our result is in complete agreement with that of Kolwankar and Gangal [31, 32]. To this aim, let us consider the inequality

$$
D_{T}<D<D_{E}
$$

where $D_{T}$ is the topological dimension and $D_{E}$ is the embedding dimension [1]. The condition (44) needs to be fulfilled by any function in order to be a fractal. In the specific case of the graph of a fractal function, $D_{T}=1$ and $D_{E}=2$. The same condition (44) must also hold true for the fractional derivative (fractional integral) of the function, in this case the GWF, in order to preserve its fractal properties. Therefore, for the fractional derivative of GWF, we have: 


$$
D^{\prime \prime}<2 \Rightarrow D+\beta<2 \Rightarrow \beta<2-D .
$$

This means that the generalized Weierstrass function is fractionally differentiable for all orders less than $2-D$, which is the result of Kolwankar and Gangal [31,32].

Moreover, the same kind of reasoning can be applied to the fractional integral of the GWF. In this case, the meaningful condition reads:

$$
D^{\prime}>1 \Rightarrow D-\beta>1 \Rightarrow \beta<D-1 .
$$

This means that the generalized Weierstrass function is fractionally integrable for all orders less than $D-1$.

Finally, we go back to the main issue, that being, a possible equation of evolution for a complex system, exhibiting fractal behavior and perhaps representable by the increments of a generalized Weierstrass function.

Consider the function

$$
f(t)=\sum_{n=-\infty}^{\infty} A_{n}\left(1-e^{i \omega_{n} t}\right) e^{i \phi_{n}}
$$

where $\left\{\omega_{n}\right\}$ is a set of frequencies, $\left\{\phi_{n}\right\}$ is a set of random phase confined to the interval $(0,2 \pi)$, and $A_{n}$ is a real amplitude. Thus, $f(t)$ is a stochastic function of time with the definite initial condition $f(0)=0$. If we now use (47) as a driving force for a fractional stochastic equation we can write

$$
\mathcal{D}^{(\alpha)} F(t)=f(t)
$$

as our equation of motion. The solution to (48) is formally given by the inverse equation

$$
F(t)=\mathcal{D}^{(-\alpha)} f(t)
$$

so that using the definition of the fractional integral, (16), we obtain the explicit form of the solution

$$
F(t)=\sum_{n=-\infty}^{\infty} \frac{A_{n}}{\omega_{n}^{\alpha}}\left(1-e^{i \omega_{n} t}\right) e^{i \phi_{n}^{\prime}}
$$

where we have absorbed the overall phase into the new random phases $\left\{\phi_{n}^{\prime}\right\}$. Therefore, if we choose the parameter values $\omega_{n}=\gamma^{n}, A_{n}=1$ and $\alpha=2-D$, the solution to the fractional stochastic differential equation, (48), is the GWF.

In addition if we choose the coefficients in the series representation of the random driving force by $A_{n} \propto 1 / \omega_{n}$ then (47) is a realization of a complex Brownian motion process, which is to say that the statistics are two dimensional Gaussian and the spectrum is an inverse square frequency. In this case if we also choose for the parameters in the solution to the fractional stochastic differential equation $\omega_{n}=\gamma^{n}$ and $\alpha=1-D$, then the solution is again a GWF. In addition, as pointed out by Mandelbrot [1] (page 390), the GWF with random coefficients is a good approximation to a fractional Brownian function.
[1] B.B. Mandelbrot, The Fractal Geometry of Nature, W.H. Freeman and Co., San Francisco (1977).

[2] B.J. West, Physiology, Promiscuity and Prophecy at the Millennium: A Tale of Tails, Studies of Nonlinear Phenomena in the Life Sciences Vol. 9, World Scientific, Singapore (1998).

[3] P. Meakin, Fractals, scaling and growth far from equilibrium, Cambridge Nonlinear Science Series 5, Cambridge University Press, Cambridge (1998).

[4] M. Schroeder, Fractals, Chaos, Power Laws, W.H. Freeman and Comp., New York (1991).

[5] L.F. Richardson, Proc. Roy. Soc. London A 110 (1926) 709.

[6] M.F. Shlesinger, B.J. West and J. Klafter, Phys. Rev. Lett. 58 (1987) 1100.

[7] P. Lévy, Calcul des probabilities, Guthier-Villars, Paris (1925); Théorie de l'addition des variables aléatoires, Guthier-Paris (1937).

[8] B.D. Hughes, Random Walks and Random Environments, Volume 1: Random Walks, Oxford Science Publications, Clarendon Press, Oxford (1995).

[9] E.W. Montroll and B.J. West, in Fluctuation Phenomena, pp. 61-206, Editors E.W. Montroll and J.L. Lebowitz, Second Edition, North-Holland Personal Library, North-Holland, Amsterdam (1987)

[10] E.W. Montroll and M.F. Shlesinger, in Nonequilibrium Phenomena II: From Stochastics to Hydrodynamics, pp. 1-121, Editors E.W. Montroll and J.L. Lebowitz, NorthHolland, Amsterdam (1983).

[11] P. Langevin, C.R. Acad. Sci. 530, Paris (1908).

[12] J. Perrin, "Mouvement brownien et réalité molé culaire", Annales de chimie et de physique VIII 18, 5-114: Translated by F. Soddy as Brownian Movement and Molecular Reality, Taylor and Francis, London.

[13] J.L. Doob, Ann. Math. 43 (1942) 351.

[14] G.E. Uhlenbeck and L.S. Ornstein, Phys. Rev. 36 (1930) 823.

[15] P. Grigolini, A. Rocco and B.J. West, to appear in Phys. Rev. E.

[16] P. Allegrini, P. Grigolini and B.J. West, Phys. Rev. E 54 (1996) 4760.

[17] A. Compte, Phys. Rev. E 53 (1996) 4191.

[18] A.S. Chaves, Phys. Lett. A 239 (1998) 13.

[19] J.T.M. Hosking, Biometrika 68 (1981) 165.

[20] J. Bernan, Statistics of Long-Memory Processes, Monograph on Statistics and Applied Probability 61, Chapman and Hall, New York (1994).

[21] B.J. West and P. Grigolini, in Applications of Fractional Calculus in Physics, Editor R. Hilfer, World Scientific, Singapore (1998).

[22] W.G. Glöckle and T.F. Nonnenmacher, Rheol. Acta 33 (1994) 337; J. Stat. Phys. 71 (1993) 741.

[23] M. Berry, J. Phys. A: Math. Gen. 12 (1979) 781.

[24] W.R. Schneider and W. Wyss, J. Math. Phys. 30 (1989) 134. 
[25] This view is not free of criticism. Many attempts have been made to reproduce the thermodynamical properties of the Universe from Newton's laws, but it seems that this can not be done in a satisfactory way unless nondynamical assumptions like coarse graining, the Markov approximation or Van Hove approaches are made [15].

[26] A.S. Monin and A.M. Yaglom, Statistical Fluid Mechanics: Mechanics of Turbulence, Volumes 1 and 2, MIT Press, Cambridge, Mass. (1971).

[27] K. Falconer, Fractal Geometry, Wiley, New York (1990).

[28] M.V. Berry and Z.V. Lewis, Proc. Roy. Soc. Lond. A 370 (1980) 459.
[29] In the following we use the term fractional dimension to avoid specious arguments over whether $D$ is the box counting dimension, the Hausdorff-Besicovitch dimension or the fractal dimension. For our immediate purposes it is sufficient that $D$ is not an integer and is in the interval $1<D<2$.

[30] R.D. Mauldin and S.C. Williams, Trans. Am. Math. Soc. 298 (1986) 793.

[31] K.M. Kolwankar and A.D. Gangal, Chaos 6 (1996) 505.

[32] K.M. Kolwankar and A.D. Gangal, Pramana J. Phys. 48 (1997) 49. 